Published in "Journal of Electromyography and Kinesiology

25(2): 199-204, 2015"

which should be cited to refer to this work.

\title{
Brachialis muscle activity can be assessed with surface electromyography
}

\author{
Didier Staudenmann*, Wolfgang Taube \\ Movement and Sport Science, Department of Medicine, Faculty of Science, University of Fribourg, 1700 Fribourg, Switzerland
}

\begin{abstract}
The brachialis muscle (BR) represents an important elbow flexor and its activity has so far mainly been measured with intramuscular electromyography (EMG). The aim of this study was to examine whether the activity of the BR can be assessed with surface EMG without interference from the biceps brachii (BB). With eight subjects we measured surface EMG of the arm flexor synergists, BR, BB, and brachioradialis (BRR) during two isometric voluntary contraction types: (1) pure elbow flexion and (2) elbow flexion with a superimposed forearm supination. Since the BR and BB have a distinct biomechanical function, an individual activity of the BR can be expected for the second contraction type, if the BR can be assessed independently from the BB. The correlation coefficients between EMG amplitudes and flexion force (supination torque) were determined. During pure flexion the activities of all synergists were similarly correlated with the flexion force $(r=0.96 \pm 0.02)$. During flexion + supination the activity of the BR was distinct from the activity of the BB, with a 14\% higher correlation for the BR with the flexion force and a $40-64 \%$ lower correlation with the supination torque. The BB predicted supination torque substantially better than the BR and BRR $(r=0.93 \pm 0.02)$. The current results demonstrate that the activity of the BR can be assessed with surface EMG as it was distinct from the BB during flexion + supination but predicted flexion force equally well as BB during the pure flexion contraction.
\end{abstract}

\section{Introduction}

The elbow flexor muscles are the brachialis (BR), biceps brachii (BB) and brachioradialis (BRR, Murray et al., 2000), with the greatest contribution to elbow flexion from the BR as shown with magnetic resonance imaging (Kawakami et al., 1994). So far, activity of this important elbow flexor has mainly be measured with intramuscular electromyography (EMG, e.g. Basmajian and Latif, 1957; Buchanan et al., 1986; Hodges et al., 2003; Naito et al., 1998; Rudroff et al., 2008; Staudenmann et al., 2009; van Bolhuis et al., 1997; van Zuylen et al., 1988) but there exist a few studies using surface EMG (e.g. Praagman et al., 2010). However, none of these studies has tested the quality of the recordings, i.e., the possible interference from adjacent muscles. Therefore, it is not surprising that the European recommendations for surface EMG recordings do not list the BR (cf. Hermens et al., 2000) as it remains still unclear whether the activity of the BR can be accurately assessed with surface EMG.

The proximal part of the BR is - similarly to the soleus muscle covered with a superficial muscle (i.e. BB), and the distal part of the

* Corresponding author at: University of Fribourg, Bd. de Pérolles 90, CH-1700 Fribourg, Switzerland. Tel.: +41263007286.

E-mail address: didier.staudenmann@unifr.ch (D. Staudenmann).
BR becomes gradually superficial (cf. Herbert and Gandevia, 1995). As the distal part of the soleus can be measured with surface EMG (Hermens et al., 2000), the distal superficial part of the BR should, in principle, also be accessible with surface EMG. Moreover, the BB and BR have different biomechanical functions. The BR inserts into the ulna and is therefore exclusively responsible for elbow flexion (cf. Leonello et al., 2007) while the BB inserts into the radius (biceps tubercle) and therefore has the particularity that it not only acts as an elbow flexor but also as forearm supinator (cf. van Zuylen et al., 1988). Accordingly, when generating a contraction in which an elbow flexion is combined with a forearm supination, a differentiation in the EMG signal between the BB and BR can be expected, provided that the signals are selective enough to capture the individual muscle activities.

The aim of this study was to examine whether the activity of one of the most important elbow flexor muscles, the BR, can be assessed by means of surface EMG without interference from the BB. For this purpose we measured all elbow flexion synergists (BB, BR and BRR) while performing two contraction types: first, a pure elbow flexion, and second, an elbow flexion with a superimposed forearm supination. We hypothesized that selective assessment of the EMG signals should result in comparable activity in the synergists in the first contraction type, while differences between the BR 
and $\mathrm{BB}$ muscles were expected in the second contraction type where flexion is combined with supination.

\section{Methods}

\subsection{Subjects and procedures}

Eight healthy men (mean \pm standard deviation: age, $35 \pm$ 11 years; mass, $71.5 \pm 6.4 \mathrm{~kg}$; height, $177 \pm 6 \mathrm{~cm}$ ), who reported no neurological or cardiovascular disorders, participated in the experiment. Informed written consent was obtained before participation in the study. The study was approved by the local ethics committee and was conducted in accordance with the latest declaration of Helsinki.

Subjects were seated upright in a chair with the right upper arm abducted to an angle of about $20^{\circ}$ with the forearm in a horizontal position midway between supination-pronation, and the elbow joint was flexed to a $90^{\circ}$ angle (Fig. 1). Before the contraction, each subject performed three maximum voluntary contractions (MVCs)

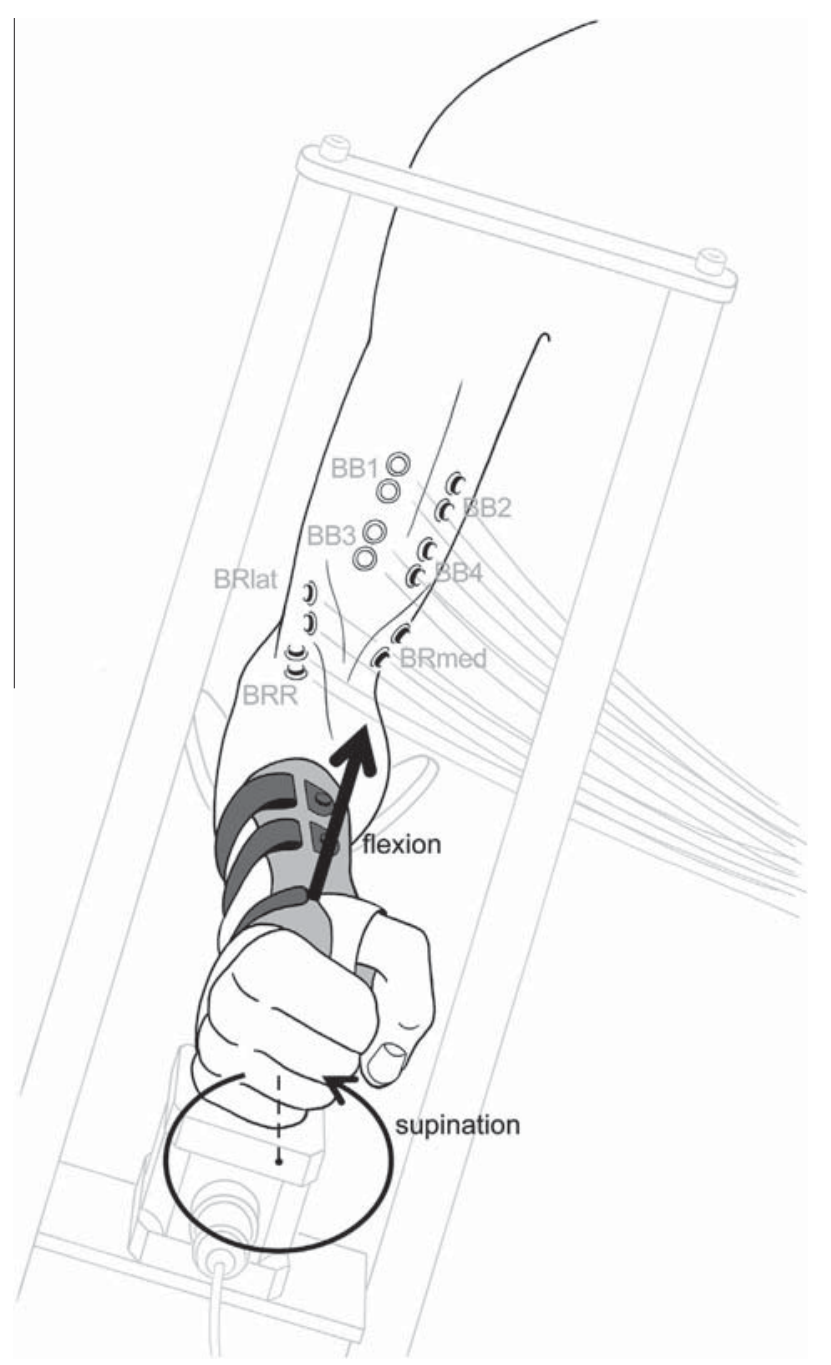

Fig. 1. Position of the right arm during the isometric elbow flexion (plus forearm supination) contraction with electrode locations over biceps brachii (BB1-4), brachialis (BRlat/med) and brachioradialis (BRR). The electrode pairs BB1-2 were located over the proximal part of the long and short head, respectively, and BB3-4 were placed over the proximal part of the corresponding two muscle heads. Note that the forearm was tightly fixed in a wrist-hand-thumb orthosis attached to the force transducer located below the wrist. The frame was aligned to the arm position in order to measure pure flexion forces. with the elbow flexor muscles by gradually increasing the force from rest to maximum, and then decreasing back to baseline. The highest value was retained as MVC. Then, two isometric contraction types had to be performed: first, a pure elbow flexion that started at baseline and was gradually increased up to $20 \%$-MVC within $2-3 \mathrm{~s}$ before it was sustained for $4-5 \mathrm{~s}$ and subsequently released back to baseline. For the second contraction type, a gradually increased supination torque was superimposed on the sustained 20\%-MVC elbow flexion. In order to control the desired flexion force intensity, a screen was located in front of the subjects displaying a horizontal line at 20\%-MVC and subjects received online feedback of the exerted flexion force.

\subsection{Mechanical and electrophysiological recordings}

Subjects had their right elbow supported on a pad that restrained arm movements. The forearm was tightly fixed in a custom-made wrist-hand-thumb orthosis (Orthopedics Ruffieux, Fribourg, Switzerland) that was attached to a force transducer (AMTI, Watertown, MA, USA) to measure the upward force and the supination-pronation torque at the wrist (Fig. 1). In order to capture a pure supination torque at the wrist, collateral forces were subtracted from the supination-pronation torque. For this purpose, a reference measurement of a pure lateral force was conducted to determine the biased torque contribution. The frame and the force transducer were aligned to the arm position so that an elbow flexion mainly produced a pure force in the upward direction (Fig. 1). The signals from the force transducer were recorded using custom-built software (LabVIEW-based, National Instruments, Austin, TX, USA, AD conversion at 2000 samples/s, 16-bit resolution).

Surface EMG was recorded from all elbow flexion synergists, BB, $\mathrm{BR}$, and BRR, using microelectrodes ( $\varnothing 2 \mathrm{~mm}$; TMSi, Oldenzaal, NL) in a bipolar configuration ( $1 \mathrm{~cm}$ interelectrode distance). Recordings of the BB were obtained from the proximal and distal portion of the two muscle heads, as distinct EMG activities of the two muscle parts were observed for specific contractions (Perot et al., 1996; Staudenmann et al., 2013; ter Haar Romeny et al., 1984; van Zuylen et al., 1988). Furthermore, the activity of the BR was recorded over the medial and lateral sides of the distal upper arm where the muscle becomes superficial, and the electrodes over the BRR were attached as displayed in Fig. 1. The reference electrode was placed over the lateral epicondyle of the elbow. The electrodes were connected to the amplifier (REFA, TMSi, Oldenzaal, NL) and processed as monopolar EMG signals (input impedance $>10^{12} \Omega$, internal anti-aliasing filter at $400 \mathrm{~Hz}, \mathrm{AD}$ conversion at 2000 samples/s, 24-bit resolution).

\subsection{Data analysis}

The data were analyzed with MATLAB (The MathWorks, Inc, Natick MA, USA). Force signals were aligned to the EMG signals with a synchronization pulse. The monopolar EMGs were highpass filtered $(10 \mathrm{~Hz}$, Butterworth, 1st order, bidirectional) and a single bipolar EMG was derived for the different recording sides. Subsequently, the EMG signals were rectified and smoothed ( $2 \mathrm{~Hz}$, Butterworth, 1st order, bidirectional) to get an EMG amplitude (cf. Fig. 2). We used correlation coefficients between EMG amplitude and flexion force (supination torque) to get an indication of the dependency between both signals.

\subsection{Statistics}

The results were analyzed with repeated measures analysis of variance (ANOVA) with the significance level set at $5 \%$. The following dependent variables were examined with 7 electrode 

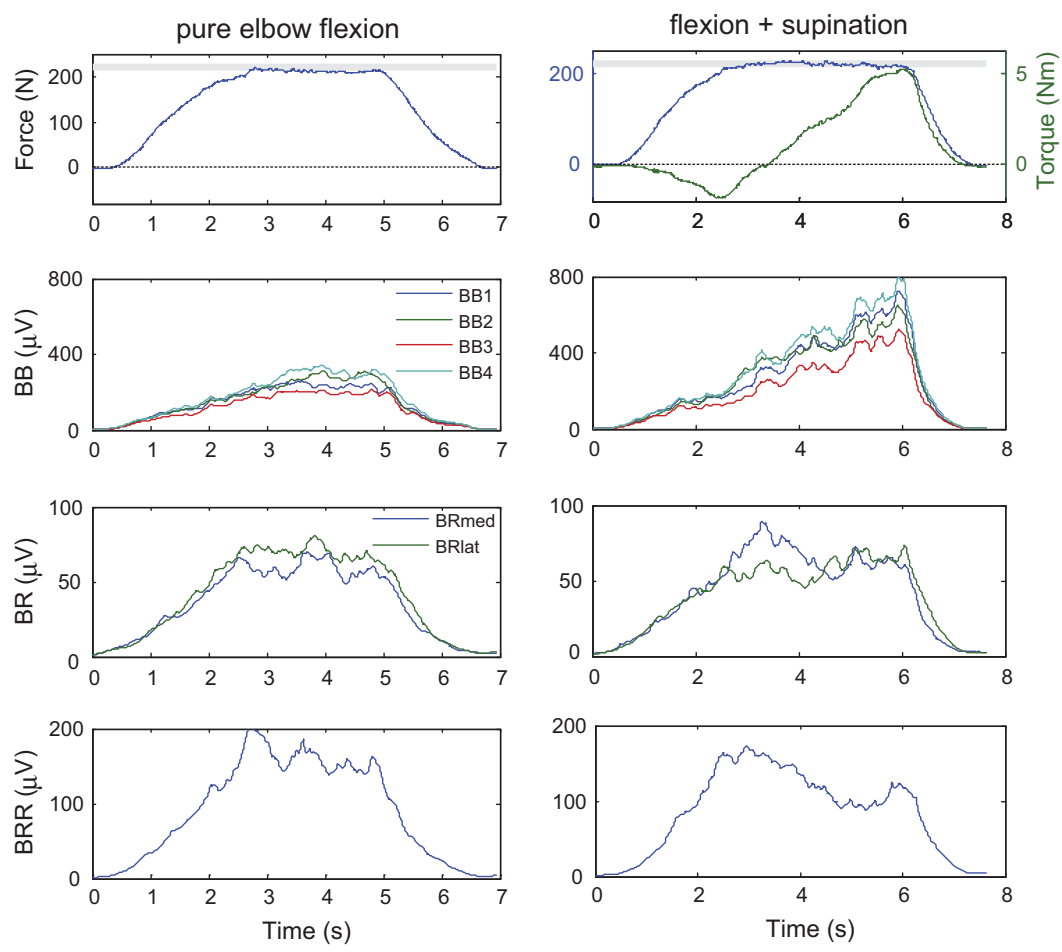

Fig. 2. Data from one representative subject performing the two contraction types (pure elbow flexion and flexion + supination). The gray bar of the upper panel shows the target flexion force at 20\%-MVC (for this subject $\sim 220 \mathrm{~N}$ ), which had to be reached and held by the subject. The lower panels show EMG amplitudes for the three arm flexion synergists. Muscular activations obtained by the 4 electrode locations over the biceps brachii (BB), the 2 locations over the brachialis (BR), and the recording sites of the brachioradialis (BRR) are displayed. Note that the BR activity is clearly distinct from the BB for the second contraction type (flexion + supination).

locations as fixed factor: Correlation coefficients (after Fisher-Z transformation) between EMG amplitude and flexion force for the first contraction type (pure elbow flexion) and between EMG amplitude and flexion force/supination torque for the second contraction type (flexion + supination). In case of significant values of the ANOVA a post hoc pairwise $t$-test with Bonferroni correction was conducted within and between muscles. Data are reported as mean \pm standard deviation.

\section{Results}

For the first contraction type (pure elbow flexion) no significant difference between electrode locations was found for the correlation between EMG amplitudes and arm flexion force $\left(F_{6,7}=2.08\right.$; $p=0.075$ ). As can be seen in Fig. 3, activities of all muscles showed a high correlation with the flexion force $(r=0.96 \pm 0.02)$. For the second contraction type (flexion + supination), the correlation between EMG amplitude and flexion force was lower (Fig. 4) and the electrode locations showed a significant difference in the correlation between EMG amplitude and flexion force $\left(F_{6,7}=7.74 ; p<0.01\right)$. The post hoc analysis between the four sides within the BB were non-significant $(p=1, r=0.72 \pm 0.09)$ and the comparison between the two sides within the BR showed only a trend towards significance (lateral: $r=0.88 \pm 0.09$, medial: $r=0.80 \pm 0.11 ; p=0.053$ ). The lateral $(+18 \% ; p=0.037)$ and the medial part of the BR $(+10 \%$; $p=0.085$ ) displayed higher correlations than the $\mathrm{BB}$ for the pure elbow flexion. Between the BRmed, BRR and BB no significant difference in the correlation between EMG amplitude and flexion force was found (all $p>0.077$ ).

For the supination torque the EMG amplitudes of the different electrode locations also showed a significant difference $\left(F_{6,7}=\right.$ 56.8; $p<0.01$; Fig. 5 ). The post hoc analysis between the four sites

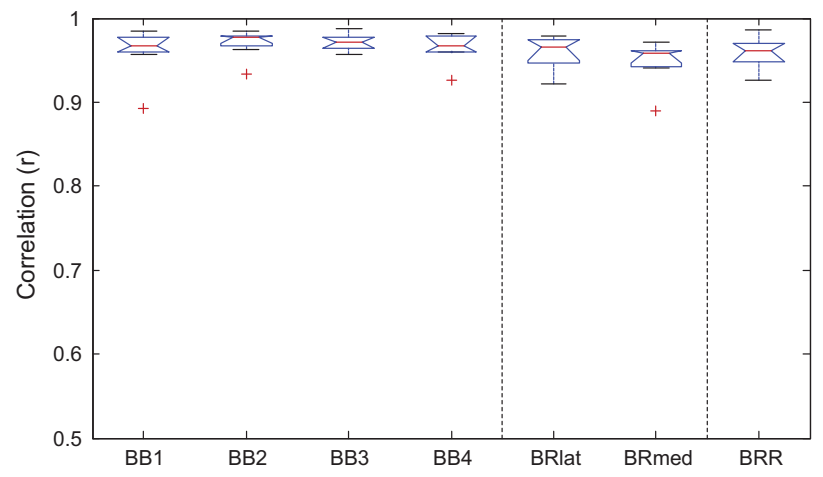

Fig. 3. Correlation between EMG amplitude and elbow flexion force for the first contraction type (pure elbow flexion). Boxplots show the results for all subjects; the middle line in each boxplot represents the median value and the error bars indicate the range. The lower and upper limits of each box represent the interquartile range, and the plus signs denote outliers. Note all synergists are equally well associated to flexion force as no significant effect was found between muscles.

within the BB was non-significant $(p>0.11)$, whereas the two sites within the BR differed significantly from each other $(p=0.046)$ with a $40 \%$ higher correlation for the lateral $(r=0.55 \pm 0.21)$ compared to the medial part $(r=0.33 \pm 0.29)$. Comparisons between muscles revealed that the averaged correlation coefficients from the BB $(r=0.93 \pm 0.02)$ were significantly higher by about $40-64 \%$ compared with the BR $(p<0.01)$. Thus, the BB predicted supination torque substantially better than the BR, which clearly shows a differentiation between these muscles when predicting supination torque. The BRR was also unrelated to the activity of the lateral BR $(p<0.01)$ and displayed the lowest correlation coefficients $(r=0.22 \pm 0.19)$ 


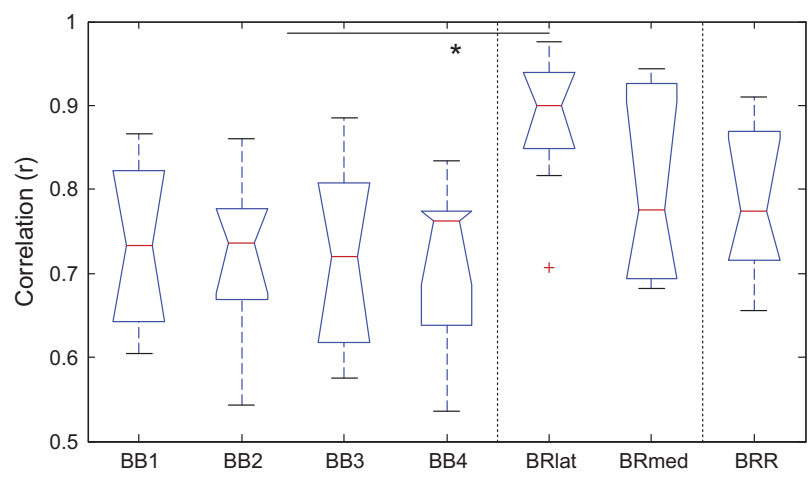

Fig. 4. Correlation between EMG amplitude and elbow flexion force for the second contraction type (flexion + supination). Boxplots show the results for all subjects; the middle line in each boxplot represents the median value and the error bars indicate the range. The lower and upper limits of each box represent the interquartile range and the plus signs denote outliers. Note that the BR shows a higher association to flexion force compared to the BB muscle.

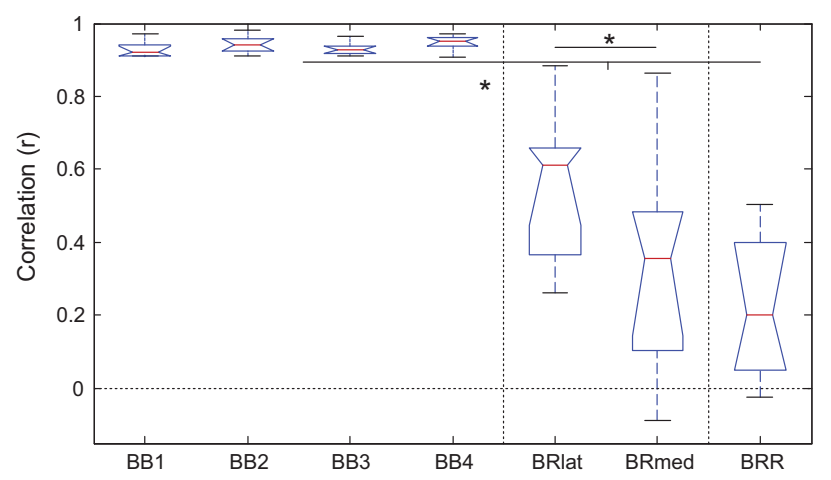

Fig. 5. Correlation between EMG amplitude and forearm supination torque for the second contraction type (flexion + supination). Boxplots show the results for all subjects; the middle line in each boxplot represents the median value and the error bars indicate the range. The lower and upper limits of each box represent the interquartile range. Note that the BB shows a higher association to supination torque compared to the BR and BRR muscles.

\section{Discussion}

BR represents one of the most important elbow flexor muscles (cf. Kawakami et al., 1994). So far, its activity was mainly measured with intramuscular EMG (e.g., Buchanan et al., 1986; Rudroff et al., 2008; van Bolhuis et al., 1997; van Zuylen et al., 1988). The few studies that have investigated the BR activity with surface EMG did not test for potential interference from adjacent muscles (e.g. Praagman et al., 2010). The aim of this study was to examine whether the activity of one of the most important elbow flexor muscles, the BR can be assessed with surface EMG. For this purpose we collected the activity of all elbow flexion synergists (BB, BR, $\mathrm{BRR}$ ) in order to compare their EMG amplitudes to the corresponding force/torque pattern during two isometric contraction types: (1) pure elbow flexion and (2) elbow flexion with a superimposed forearm supination. These two contraction types were chosen in order to verify whether the activity of BR is distinct from that of the $\mathrm{BB}$ as these muscles have different biomechanical functions based on their anatomy. The BB inserts into the radius (biceps tubercle) and is thus active during elbow flexion and forearm supination, while the BR inserts into the ulna and therefore exclusively contributes to flexion force (cf. Leonello et al., 2007). As the activity of the BR on the one hand corresponded equally well to the exerted flexion force (see Fig. 3), but on the other hand differed significantly from the activity of the BB during superimposed supination (see Figs. 3 and 4), it can be concluded that the muscular activity of the BR can be assessed with surface EMG recordings without substantial interference from the BB.

The muscles generating an elbow flexion are the BB, BR and BRR, with a major contribution from the BR (Kawakami et al., 1994). In the current study, activities in these synergistic muscles were similarly well correlated to flexion force in the first contraction type ( $r=0.96 \pm 0.02$; see Fig. 3$)$. Nevertheless, it might be argued that the activity of the BR was contaminated with crosstalk from the adjacent BB muscle. However, this interpretation can be rejected, as the second contraction type (flexion + supination) displayed a clear distinction between the two muscles. BR showed a 10-14\% higher correlation than the BB to flexion force and a $40-$ $76 \%$ lower correlation to supination torque (see Figs. 4 and 5). Thus, the results of the current study show that the activity of the BR can be captured independently from that of the BB. As BB showed the highest correlation coefficients with forearm supination $(r=0.93 \pm$ 0.02; see Fig. 5) the distinct activations between the BR and the BB corresponds well to the biomechanical functions of these muscles, where the BR is exclusively responsible for elbow flexion while the $\mathrm{BB}$ also contributes to forearm supination.

When comparing the second to the first contraction type, the correlation of the BR to flexion force diminished by about $12 \%$ (see Figs. 3 and 4). This diminution is probably caused by the activity of the antagonistic muscle triceps brachii that also affects the elbow moment (cf. Murray et al., 2000) and becomes more active during forearm supination to counteract the flexion component from the BB (Hebert et al., 1991; van Zuylen et al., 1988). This phenomenon can also be observed in the current results, in which the peak in the BB activity was about twice as high when comparing the second to the first contraction type (see Fig. 2), while the activity of the BR had about the same intensity (cf. Buchanan et al., 1989). The BRR showed a slight reduction in the second contraction type, possibly due to reciprocal inhibition from the higher BB activity (cf. Naito et al., 1998). However, although the BRR has the lowest force capacity among the flexor synergists (Kawakami et al., 1994), it has the largest muscular moment arm (Murray et al., 2000). This might be the reason why the BRR estimated flexion force equally well as the BB and BR (see Fig. 3).

In the second contraction type (flexion + supination), the lateral BR showed a higher correlation than the medial part. We found $9 \%$ difference between the BR sides for flexion force (see Fig. 4) and $40 \%$ difference between sides for the supination torque (see Fig. 5). When considering the results of Fig. 4, which displays closer correlation coefficients between the medial BR and BB for the flexion force, one may argue that the electrodes over the medial $\mathrm{BR}$ might be affected by crosstalk from the BB. However, as the association between EMG amplitude and supination torque (see Fig. 5) showed the opposite effect - that is to say that the lateral BR had closer correlation coefficients to the BB than the medial part - a significant contamination from the $\mathrm{BB}$ can be excluded but rather indicates that surface EMG is able to assess the BR activity independently from the $\mathrm{BB}$. This strengthens the interpretation that the differences in correlation for the lateral and the medial part of the BR might be related to distinct functional parts within the $B R$. It has indeed been shown that the BR is composed of two heads, a larger superficial and a smaller deep head, both attaching to the ulnar tuberosity on the distal side to generate forearm flexion (cf. Leonello et al., 2007). In that respect the electrodes over the lateral BR might have captured more active motor units of the larger head and therefore the EMG activity might have shown a higher correlation to flexion force.

Another observation that strengthens the fact that surface EMG can successfully capture the BR activity independently from the BB is the finding that the correlation coefficients of the BB electrodes that were closest to the two sites of the BR (BB3-BRlat and 
BB4-BRmed, see Fig. 1) did not show more similar values than the coefficients to the more distant electrodes. When regarding the activity of the four sites within the BB no significant difference between these four sites in the association to elbow flexion or forearm supination was apparent (see Figs. 3-5), although previous studies using intramuscular or multichannel EMG recordings indicated an inhomogeneous activity during the same type of contraction as in the current study (Perot et al., 1996; Staudenmann et al., 2013; ter Haar Romeny et al., 1984; van Zuylen et al., 1988). This discrepancy may be related to the limitation of conventional bipolar EMG which cannot extract muscular activity with the same precision as multichannel EMG (Staudenmann et al., 2010, 2013).

A general limitation when using surface EMG is the representativeness of the active motor units within the surface EMG (cf. Staudenmann et al., 2010). The electrodes over BR could only be placed over the collateral distal superficial portion of the muscle, thus it might be that these electrodes were not fully representative of the entire muscle activity of the BR. In that respect, it is remarkable that the BR activity represented flexion force equally as well as the $\mathrm{BB}$ and $\mathrm{BRR}$ for the first contraction type (pure elbow flexion). This indicates that the electrode location allowed a representative EMG signal of the BR activity to be assessed, resulting in a considerable estimation of the isometric force $(r=0.96 \pm 0.02$; see Fig. 3). The present results demonstrate that the activity of the $B R$ can be independently measured from the activity of the BB. However, a further limitation might be related to the relatively low contraction intensity of 20\%-MVC. We have chosen this low contraction strength in order to ensure the realization of a superimposed and gradual increase in supination torque. However, it cannot be excluded that the BR activity may be less well distinguishable at higher intensity contractions. Nevertheless, as an independent activity was found for the second contraction type (flexion + supination), in which the activity of the BB was about twice as high as during the first contraction type (see Fig. 2), it is assumed that the BR activity should also be independent of the BB activity when higher-intensity contractions are performed. A final limitation in the current study might be the relatively small sample size and that we only measured male subjects. As the outcome of the current study is clear and consistent across subjects we think that a larger sample size would not have influenced the overall finding. Furthermore, although it seems unlikely that women utilize the elbow flexor and supinator in a different way, it remains to be shown that this type of electrode positioning can assess BR activity equally well in female subjects.

Based on our results it can be concluded that the muscular activity of the BR can be assessed with surface EMG recordings without substantial interference from the BB. This was convincingly demonstrated for low contraction intensities as the activity of the BR corresponded well to the flexion force but was distinct from the activity of the BB when superimposing a supination to the flexion force.

\section{Conflict of interest}

The authors of the manuscript entitled Brachialis muscle activity can be assessed with surface electromyography declare that no financial and personal relationships with other people or organizations have inappropriately influenced the content of the work reported in this paper.

\section{Acknowledgments}

We would like to acknowledge Christoph Mayer for his contribution to the experiment and Gion Capeder for the illustration of the first figure.

\section{References}

Basmajian JV, Latif A. Integrated actions and functions of the chief flexors of the elbow. J Bone Joint Surg 1957;39-A:1106-81.

Buchanan TS, Almdale DP, Lewis JL, Rymer WZ Characteristics of synergic relation during isometric contractions of human elbow muscles. J Neurophysiol 1986;56:1225-41.

Buchanan TS, Rovai GP, Rymer WZ. Strategies for muscle activation during isometric torque generation at the human elbow. J Neurophysiol 1989;62:1201-12.

Hebert LJ, De Serres SJ, Arsenault AB. Cocontraction of the elbow muscles during combined tasks of pronation-flexion and supination-flexion. Electromyogr Clin Neurophysiol 1991;31:483-8.

Herbert RD, Gandevia SC. Changes in pennation with joint angle and muscle torque: in vivo measurements in human brachialis muscle. J Physiol 1995;484:523-32.

Hermens HJ, Freriks B, Disselhorst-Klug C, Rau G. Development of recommendations for SEMG sensors and sensor placement procedures. J Electromyogr Kinesiol 2000;10:361-74.

Hodges PW, Pengel LH, Herbert RD, Gandevia SC. Measurement of muscle contraction with ultrasound imaging. Muscle Nerve 2003;27:682-92.

Kawakami Y, Nakazawa K, Fujimoto T, Nozaki D, Miyashita M, Fukunaga T. Specific tension of elbow flexor and extensor muscles based on magnetic resonance imaging. Eur J Appl Physiol Occup Physiol 1994;68:139-47.

Leonello DT, Galley IJ, Bain GI, Carter CD. Brachialis muscle anatomy. A study in cadavers. J Bone Joint Surg Am 2007:89:1293-7.

Murray WM, Buchanan TS, Delp SL. The isometric functional capacity of muscles that cross the elbow. J Biomech 2000;33:943-52.

Naito A, Shindo M, Miyasaka T, Sun YJ, Momoi H, Chishima M. Inhibitory projections from pronator teres to biceps brachii motoneurones in human. Exp Brain Res 1998;121:99-102.

Perot C, Andre L, Dupont L, Vanhoutte C. Relative contributions of the long and short heads of the biceps brachii during single or dual isometric tasks. J Electromyogr Kinesiol 1996;6:3-11.

Praagman M, Chadwick EK, van der Helm FC, Veeger HE. The effect of elbow angle and external moment on load sharing of elbow muscles. J Electromyogr Kinesiol 2010;20:912-22.

Rudroff T, Staudenmann D, Enoka RM. Electromyographic measures of muscle activation and changes in muscle architecture of human elbow flexors during fatiguing contractions. J Appl Physiol 2008;104:1720-6.

Staudenmann D, Rudroff T, Enoka RM. Pronation-supination torque and associated electromyographic activity varies during a sustained elbow flexor contraction but does not influence the time to task failure. Muscle Nerve 2009;40:231-9.

Staudenmann D, Roeleveld K, Stegeman DF, van Dieen JH. Methodological aspects of SEMG recordings for force estimation - a tutorial and review. J Electromyogr Kinesiol 2010:20:375-87.

Staudenmann D, Stegeman DF, van Dieen JH. Redundancy or heterogeneity in the electric activity of the biceps brachii muscle? Added value of PCA-processed multi-channel EMG muscle activation estimates in a parallel-fibered muscle. J Electromyogr Kinesiol 2013;23:892-8.

ter Haar Romeny BM, van der Gon JJ, Gielen CC. Relation between location of a motor unit in the human biceps brachii and its critical firing levels for different tasks. Exp Neurol 1984;85:631-50.

van Bolhuis BM, Medendorp WP, Gielen CC. Motor unit firing behavior in human arm flexor muscles during sinusoidal isometric contractions and movements. Exp Brain Res 1997;117:120-30.

van Zuylen EJ, Gielen CC, Denier van der Gon JJ. Coordination and inhomogeneous activation of human arm muscles during isometric torques. J Neurophysiol 1988;60:1523-48. 